\title{
Disturbance Attraction Domain Estimation for Saturated Markov Jump Systems with Truncated Gaussian Process
}

\author{
Yanqing Liu, ${ }^{1,2}$ Yanyan Yin, ${ }^{1}$ Fei Liu, ${ }^{1}$ and Kok Lay Teo ${ }^{2}$ \\ ${ }^{1}$ Key Laboratory of Advanced Process Control for Light Industry, Ministry of Education, Institute of Automation, \\ Jiangnan University, Wuxi 214122, China \\ ${ }^{2}$ Department of Mathematics and Statistics, Curtin University, Perth, WA 6102, Australia
}

Correspondence should be addressed to Fei Liu; fliu@jiangnan.edu.cn

Received 9 December 2013; Revised 4 April 2014; Accepted 4 April 2014; Published 24 April 2014

Academic Editor: Xiaojie Su

Copyright (C) 2014 Yanqing Liu et al. This is an open access article distributed under the Creative Commons Attribution License, which permits unrestricted use, distribution, and reproduction in any medium, provided the original work is properly cited.

\begin{abstract}
This paper investigates the disturbance attraction domain estimation of saturated Markov jump systems with truncated Gaussian process. The aim is to estimate the disturbance domain of attraction so that the state is maintained in a neighbour around the origin by a state feedback controller regardless of bounded disturbance. The problem is formulated as parameter-dependent linear matrix inequalities (LMIs). The optimal disturbance attraction domain is obtained through searching for most appropriate auxiliary parameters in the defined domain. A numerical example is presented to show the potential application of the results.
\end{abstract}

\section{Introduction}

For a system subject to abrupt structural changes, such as component failures and sudden environmental changes, it is more appropriate to model it as a Markov jump linear system (MJS), where the switching behaviour amongst the different modes of the system is determined by its transition probability (TP) governed by a finite Markov chain. Many results related to controller design under the time-invariant transition probability are now available in the literature (see, e.g., [1-11] and the references therein). However, the exact value of the transition probability cannot be easily obtainable. It is often that only partial information of the transition probability can be obtained. In this situation, questions on the stability analysis and controller design (see [12-14]) have also been addressed. In practice, the environment can be so complex that the transition probability of the MJS concerned can only be nonhomogeneous. For example, the delay and packet loss of a networked control system are distinct among different working time [15]. Similar phenomena are also observed in electronic circuits [16] and manpower systems [17]. For Markov systems with nonhomogeneous transition probability, some interesting results are now available (see [18, 19]). In [20], a new method for describing the time-varying transition probability in the statistic sense is proposed. This approach covers the cases where the transition probabilities are known either exactly or partially as special cases.

On the other hand, saturation failure is widely encountered in engineering applications. In the presence of saturation nonlinearity, a linear system will become a highly complex nonlinear system [21]. It is well known that nonlinear systems do not have, in general, global stability property [22]. Thus, the problem of attraction domain estimation has become a fundamentally challenging problem in nonlinear control theory [23]. For a linear system with saturation, some results related to attraction domain estimation have been obtained (see, e.g., [24, 25]). However, it appears that the estimation of the attraction domain for a saturated Markov system with nonhomogeneous transition probability has not been fully investigated. The situation will become much worse when there is disturbance to the system, as the behavior of the system will be significantly degraded by disturbance. The difficulties mentioned above are the motivation behind this paper to study the disturbance attraction domain estimation for discrete-time Markov jump systems with saturation and subject to truncated Gaussian transition probability. Based on [20], the aim of this paper is to propose a novel approach to estimate the optimal domain of attraction which can restrain the states of system to be within the smallest neighborhood around the origin under the bounded disturbance. 
The rest of the paper is organized as follows: in Section 2, the system is defined, Section 3 introduces the concept of stochastic stability, in Section 4, sufficient conditions for disturbance attraction domain estimation are derived, in Section 5, a numerical example is provided to illustrate the applicability of the results obtained, and Section 6 concludes the paper.

In the sequel, the notation $R^{n}$ stands for an $n$-dimensional Euclidean space; the transpose of the matrix $A$ is denoted by $A^{\mathrm{T}} ; E\{\cdot\}$ denotes the mathematical statistical expectation of the stochastic process or vector; $\partial$ is the boundary of a set; a positive-definite matrix is denoted by $P>0$; $I$ is the unit matrix with appropriate dimension; and $*$ means the symmetric term in a symmetric matrix.

\section{Problem Statement and Preliminaries}

Let $(M, F, P)$ be a probability space, where $M, F$, and $P$ represent, respectively, the sample space, the $\sigma$-algebra of events, and the probability measure defined on $F$. Consider the following discrete-time Markov jump system:

$$
x_{k+1}=A\left(r_{k}\right) x_{k}+B\left(r_{k}\right) \sigma\left(u_{k}\right)+E\left(r_{k}\right) w_{k},
$$

where $x_{k} \in R^{n}$ is the state, $u_{k} \in R^{m}$ is the input, $w_{k} \in$ $\left\{w_{k}^{\mathrm{T}} w_{k} \leq 1\right\}$ is the bounded disturbance of the system, and $\sigma\left(u_{k}\right)=\left[\begin{array}{llll}\sigma\left(u_{1 k}\right) & \sigma\left(u_{2 k}\right) & \cdots & \sigma\left(u_{m k}\right)\end{array}\right]^{\mathrm{T}}$.

The system is driven by a random process $\left\{r_{k}, k \geq 0\right\}$ which takes values from a finite set $\Gamma=\{1,2,3, \ldots, s\}$, where $\pi_{r_{k} r_{k+1}}^{\left(\xi_{k}\right)}=\operatorname{Pr}\left(r_{k+1}=j \mid r_{k}=i, \xi_{k}\right)$ denotes the transition probability from mode $i$ at time $k$ to mode $j$ at time $k+1$. Here, it is assumed that the TP, which is nonhomogeneous, is approximated by a set of random variables driven by a truncated Gaussian stochastic process $\left\{\xi_{k}, k \geq 0\right\}$. The probability density function (PDF) of $\pi_{r_{k} r_{k+1}}^{\left(\xi_{k}\right)}$ is given as follows:

$$
\pi_{r_{k} r_{k+1}}^{\left(\xi_{k}\right)}=\frac{\left(1 / \sigma_{r_{k} r_{k+1}}\right) f\left(\left(\pi_{r_{k} r_{k+1}}^{\left(\xi_{k}\right)}-\mu_{r_{k} r_{k+1}}\right) / \sigma_{r_{k} r_{k+1}}\right)}{F\left(\left(1-\mu_{r_{k} r_{k+1}}\right) / \sigma_{r_{k} r_{k+1}}\right)-F\left(\left(0-\mu_{r_{k} r_{k+1}}\right) / \sigma_{r_{k} r_{k+1}}\right)},
$$

where $f(\cdot)$ is the PDF of the standard normal distribution, $F(\cdot)$ is the cumulative density function (CDF) of $f(\cdot)$, and $\mu_{r_{k} r_{k+1}}$ and $\sigma_{r_{k} r_{k+1}}^{2}$ are, respectively, the mean and variance of the Gaussian PDF. More specifically, the TP matrix is given by

$$
\pi=\left[\begin{array}{cccc}
n\left(\mu_{11}, \sigma_{11}^{2}\right) & n\left(\mu_{12}, \sigma_{12}^{2}\right) & \ldots & n\left(\mu_{1 s}, \sigma_{1 s}^{2}\right) \\
n\left(\mu_{21}, \sigma_{21}^{2}\right) & n\left(\mu_{22}, \sigma_{22}^{2}\right) & \ldots & n\left(\mu_{2 s}, \sigma_{2 s}^{2}\right) \\
\vdots & \vdots & \ddots & \vdots \\
n\left(\mu_{s 1}, \sigma_{s 1}^{2}\right) & n\left(\mu_{s 2}, \sigma_{s 2}^{2}\right) & \ldots & n\left(\mu_{s s}, \sigma_{s s}^{2}\right)
\end{array}\right],
$$

where $n\left(\mu_{r_{k} r_{k+1}}, \sigma_{r_{k} r_{k+1}}^{2}\right)$ denotes the PDF of truncated Gaussian TP of $p\left(\pi_{r_{k} r_{k+1}}^{\left(\xi_{k}\right)}\right)$, which is assumed to be known a priori.

It is noted that a larger $\sigma^{2}$ implies a larger degree of uncertainty related to the TP. In this case, a larger $\sigma^{2}$ should be chosen. Otherwise, a smaller $\sigma^{2}$ should be chosen. The random variables $\pi_{r_{k} r_{k+1}}^{\left(\xi_{k}\right)}$ which appeared in the TP matrix are continuous. Taking the expectation of the random variable yields

$$
\begin{aligned}
& \widehat{\pi}_{r_{k} r_{k+1}}^{\left(\xi_{k}\right)} \\
& =E\left(\pi_{r_{k} r_{k+1}}^{\left(\xi_{k}\right)}\right) \\
& =\int_{0}^{1} \pi_{r_{k} r_{k+1}}^{\left(\xi_{k}\right)} p\left(\pi_{r_{k} r_{k+1}}^{\left(\xi_{k}\right)}\right) d \pi_{r_{k} r_{k+1}}^{\left(\xi_{k}\right)} \\
& =\mu_{r_{k} r_{k+1}} \\
& \quad+\frac{f\left(\left(1-\mu_{r_{k} r_{k+1}}\right) / \sigma_{r_{k} r_{k+1}}\right)-f\left(\left(0-\mu_{r_{k} r_{k+1}}\right) / \sigma_{r_{k} r_{k+1}}\right)}{F\left(\left(1-\mu_{r_{k} r_{k+1}}\right) / \sigma_{r_{k} r_{k+1}}\right)-F\left(\left(0-\mu_{r_{k} r_{k+1}}\right) / \sigma_{r_{k} r_{k+1}}\right)} \sigma_{r_{k} r_{k+1}} .
\end{aligned}
$$

Consequently, the desired TP matrix can be obtained as follows:

$$
\Pi=\left[\begin{array}{cccc}
\widehat{\pi}_{11}^{\left(\xi_{k}\right)} & \widehat{\pi}_{12}^{\left(\xi_{k}\right)} & \ldots & \widehat{\pi}_{1 s}^{\left(\xi_{k}\right)} \\
\hat{\pi}_{21}^{\left(\xi_{k}\right)} & \widehat{\pi}_{22}^{\left(\xi_{k}\right)} & \ldots & \widehat{\pi}_{2 s}^{\left(\xi_{k}\right)} \\
\vdots & \vdots & \ddots & \vdots \\
\hat{\pi}_{s 1}^{\left(\xi_{k}\right)} & \hat{\pi}_{s 2}^{\left(\xi_{k}\right)} & \ldots & \widehat{\pi}_{s s}^{\left(\xi_{k}\right)}
\end{array}\right],
$$

where $\sum_{j}^{s} \widehat{\pi}_{r_{k} r_{k+1}}^{\left(\xi_{k}\right)}=1, \hat{\pi}_{r_{k} r_{k+1}}^{\left(\xi_{k}\right)} \geq 0,1 \leq i$, and $j \leq s$.

To proceed further, we need some preliminaries.

Definition 1. Discrete-time Markov jump system (1) (with $\left.w_{k}=0\right)$ is said to be stochastically stable if

$$
\lim _{\mathrm{T} \rightarrow \infty} E\left\{\sum_{k=0}^{\mathrm{T}} x_{k}^{\mathrm{T}} x_{k} \mid x_{0}, r_{0}\right\}<\infty .
$$

Definition 2. Consider system (1); let $h_{q i}$ denote the $q$ th row of matrix $H_{i}$. Then

$$
\Theta\left(H_{i}\right)=\left\{x_{k} \in R^{n}:\left|h_{q i} x_{k}\right| \leq 1, q=1,2, \ldots, m\right\}
$$

is a symmetric polyhedron set.

Lemma 3 (see [24]). Given matrices $u_{k} \in R^{m}$ and $v_{k} \in R^{m}$ for system (1), if $\left|v_{k}\right|<1$, then $\sigma\left(u_{k}\right)=\sum_{t=1}^{2^{m}} \theta_{t}\left(M_{t} u_{k}+M_{t}^{-} v_{k}\right)$, where $0 \leq \theta_{t} \leq 1, \sum_{t=1}^{2^{m}} \theta_{t}=1, M_{t}$, and $t=1, \ldots, 2^{m}$ are $m \times m$ diagonal matrices whose diagonal elements are either 1 or 0 , and $M_{t}^{-}=I-M_{t}$.

Lemma 4 (see [24]). Given matrices $v_{k}=H_{i} x_{k}$ for system (1), if $x_{k} \in \Theta\left(H_{i}\right)$, that is $\left|v_{k}\right|<1$, then $\sigma\left(F_{i} x_{k}\right)=\sum_{t=1}^{2^{m}} \theta_{t}\left(M_{t} F_{i}+\right.$ $\left.M_{t}^{-} H_{i}\right) x_{k}$.

Definition 5. For given symmetric matrices $P_{i}>0$, let us define a mode-dependent ellipsoid invariant set given below:

$$
\varepsilon\left(P_{i}, 1\right)=\left\{x_{k} \in R^{n}: x_{k}^{\mathrm{T}} P_{i} x_{k} \leq 1\right\} .
$$




\section{Estimation of the Attraction Domain}

We first derive the sufficient condition for the estimation of the attraction domain for the case without disturbance. For simplicity, we assume that the mode at time instant $k$ is $r_{k}=i$ and the mode at time instant $k+1$ is $r_{k+1}=j$.

Theorem 6. Consider system (1) with nonhomogeneous TP matrix (5) under the condition $w_{k}=0$. Suppose that there exist a set of symmetric positive definite matrices $P_{i}>0$ and $F_{i}, H_{i}$, $\forall i \in \Gamma$, such that

$$
\begin{aligned}
& \left(A_{i}+B_{i}\left(D_{t} F_{i}+D_{t}^{-} H_{i}\right)\right)^{T} \sum_{j \in \Gamma} \widehat{\pi}_{i j} P_{j}\left(A_{i}+B_{i}\left(D_{t} F_{i}+D_{t}^{-} H_{i}\right)\right) \\
& \quad-P_{i}<0, \quad t \in\left[1,2^{m}\right]
\end{aligned}
$$$$
\varepsilon\left(P_{i}, 1\right) \subset \Theta\left(H_{i}\right) .
$$

Then the set $\cap_{i=1}^{s} \varepsilon\left(P_{i}, 1\right)$ is the domain of attraction of the closed-loop system (1).

Proof. Construct a potential Lyapunov function as

$$
V\left(x_{k}, r_{k}=i\right)=x_{k}^{\mathrm{T}} P_{i} x_{k} \quad(i \in \Gamma) .
$$

For system (1), it follows from Lemmas 3 and 4 that

$$
\begin{aligned}
& \Delta V\left(x_{k}, i\right) \\
& =E\left\{V\left(x_{k+1}, j\right)\right\}-V\left(x_{k}, i\right) \\
& =x_{k+1}^{\mathrm{T}} \sum_{j \in \Gamma} \hat{\pi}_{i j} P_{j} x_{k+1}-x_{k}^{\mathrm{T}} P_{i} x_{k} \\
& =x_{k}^{\mathrm{T}}\left[\left(A_{i}+B_{i}\left(D_{t} F_{i}+D_{t}^{-} H_{i}\right)\right)^{\mathrm{T}}\right. \\
& \left.\quad \times \sum_{j \in \Gamma} \hat{\pi}_{i j} P_{j}\left(A_{i}+B_{i}\left(D_{t} F_{i}+D_{t}^{-} H_{i}\right)\right)-P_{i}\right] x_{k} \\
& =x_{k}^{\mathrm{T}} \Phi_{i}(t) x_{k}, \quad t \in\left[1,2^{m}\right] .
\end{aligned}
$$

Clearly, condition (9) implies

$$
\Delta V\left(x_{k}, i\right)<0 .
$$

Denote $\delta=\min _{t} \lambda_{\min }\left(-\Phi_{i}(t)\right)$, for all $i \in \Gamma$, where $\lambda_{\text {min }}\left(-\Phi_{i}(t)\right)$ is the minimal eigenvalue of $\left(-\Phi_{i}(t)\right)$.

Hence,

$$
\Delta V\left(x_{k}, i\right) \leq-\delta x_{k}^{\mathrm{T}} x_{k} .
$$

Taking the sum on both sides from 0 to $\mathrm{T}$ gives

$$
\begin{aligned}
E\left\{\sum_{k=0}^{\mathrm{T}} \Delta V\left(x_{k}, i\right)\right\}= & E\left\{V\left(x_{\mathrm{T}+1}, \mathrm{~T}+1\right)\right\} \\
& -V\left(x_{0}, r_{0}\right) \leq-\delta E\left\{\sum_{k=0}^{\mathrm{T}} x_{k}^{\mathrm{T}} x_{k}\right\},
\end{aligned}
$$

which implies

$$
\lim _{\mathrm{T} \rightarrow \infty} E\left\{\sum_{k=0}^{\mathrm{T}} x_{k}^{\mathrm{T}} x_{k}\right\} \leq \frac{1}{\delta} V\left(x_{0}, r_{0}\right)<\infty .
$$

This completes the proof. Clearly Theorem 6 implies stochastic stability (see Definition 1).

\section{Estimation of Disturbance Attraction Domain}

In this section, we will derive sufficient condition for the estimation of the attraction domain under bounded disturbance. This sufficient condition will ensure that the influence of disturbance is minimized. To move forward, we assume that the bounded disturbance satisfies $w_{k}^{\mathrm{T}} w_{k} \leq 1$.

Theorem 7. Consider system (1) with nonhomogeneous TP matrix (5); suppose that there exist symmetric positive definite matrices $P_{i}>0$, and $F_{i}, H_{i}$, for all $i \in \Gamma$, such that

$\min \alpha$,

$$
\varepsilon\left(P_{i}, 1\right) \subset \alpha \chi_{\infty}
$$

$$
\begin{gathered}
\left(A_{i}+B_{i}\left(D_{t} F_{i}+D_{t}^{-} H_{i}\right)\right)^{T} \sum_{j \in \Gamma} \widehat{\pi}_{i j} P_{j}\left(A_{i}+B_{i}\left(D_{t} F_{i}+D_{i}^{-} H_{i}\right)\right) \\
+\frac{1}{1+\eta}\left(\frac{1+\eta}{\eta} \lambda_{\max }\left(E_{i}^{T} P_{j} E_{i}\right)-1\right) P_{i}<0 \\
t \in\left[1,2^{m}\right] \\
\left|h_{i q} x\right| \leq 1, \quad \forall x \subset \cap \varepsilon\left(P_{i}, 1\right), i \in \Gamma, q \in[1, m]
\end{gathered}
$$

where $\chi_{0}$ is a reference set, $x_{0}$ is an initial state, and $\alpha>0$ is a scalar; then the subset $\cap_{i=1}^{\tau} \varepsilon\left(P_{i}, 1\right)$ is the disturbance attraction domain for system (1) which satisfies an optimal disturbance attenuation performance index $\alpha$.

Proof. Consider a candidate Lyapunov function $V(x)=$ $x_{k}^{\mathrm{T}} P_{i} x_{k}$. It is required to show that

$$
\begin{aligned}
\Delta V_{k}=x_{k}^{\mathrm{T}}[ & \left(A_{i}+B_{i}\left(\sigma\left(F_{i} x\right)\right)+E_{i} w_{k}\right)^{\mathrm{T}} \\
& \left.\times \sum_{j \in \Gamma} \hat{\pi}_{i j} P_{j}\left(A_{i}+B_{i}\left(\sigma\left(F_{i} x\right)\right)+E_{i} w_{k}\right)\right] x_{k} \\
& -x_{k}^{\mathrm{T}} P_{i} x_{k}<0 .
\end{aligned}
$$


Noting that $(a+b)^{\mathrm{T}}(a+b) \leq(1+\eta) a^{\mathrm{T}} a+(1+(1 / \eta)) b^{\mathrm{T}} b$ and $w_{k}^{\mathrm{T}} w_{k} \leq 1$, it follows that

$$
\begin{aligned}
\left(A_{i}+\right. & \left.B_{i}\left(\sigma\left(F_{i} x\right)+E_{i} w_{k}\right)\right)^{\mathrm{T}} \\
& \times \sum_{j \in \Gamma} \hat{\pi}_{i j} P_{j}\left(A_{i}+B_{i}\left(\sigma\left(F_{i} x\right)+E_{i} w_{k}\right)\right) \\
\leq & \max _{t \in\left[1,2^{m}\right]} x_{k}^{\mathrm{T}}(1+\eta)\left(A_{i}+B_{i}\left(D_{t} F_{i}+D_{t}^{-} H_{i}\right)\right)^{\mathrm{T}} \\
& \times \sum_{j \in \Gamma} \hat{\pi}_{i j} P_{j}\left(A_{i}+B_{i}\left(D_{t} F_{i}+D_{t}^{-} H_{i}\right)\right) x_{k} \\
& +\left(1+\frac{1}{\eta}\right) w_{k}^{\mathrm{T}} E_{i}^{\mathrm{T}} \sum_{j \in \Gamma} \widehat{\pi}_{i j} P_{j} E_{i} w_{k}-x_{k}^{\mathrm{T}} P_{i} x_{k} \\
\leq & \max _{t \in\left[1,2^{m}\right]} x_{k}^{\mathrm{T}}(1+\eta)\left(A_{i}+B_{i}\left(D_{t} F_{i}+D_{t}^{-} H_{i}\right)\right)^{\mathrm{T}} \\
& \times \sum_{j \in \Gamma} \hat{\pi}_{i j} P_{j}\left(A_{i}+B_{i}\left(D_{t} F_{i}+D_{t}^{-} H_{i}\right)\right) x_{k} \\
& +\left(1+\frac{1}{\eta}\right) \lambda_{\max }\left(E_{i}^{\mathrm{T}} P_{j} E_{i}\right)-x_{k}^{\mathrm{T}} P_{i} x_{k} .
\end{aligned}
$$

To guarantee the attraction domain property for $x_{k} \in$ $\cap \varepsilon\left(P_{i}, 1\right)$, it suffices to show that there exists an $\eta$, for all $t \in\left[1,2^{m}\right]$ such that

$$
\begin{aligned}
& x_{k}^{\mathrm{T}}(1+\eta)\left(A_{i}+B_{i}\left(D_{t} F_{i}+D_{t}^{-} H_{i}\right)\right)^{\mathrm{T}} \\
& \quad \times \sum_{j \in \Gamma} \hat{\pi}_{i j} P_{j}\left(A_{i}+B_{i}\left(D_{t} F_{i}+D_{t}^{-} H_{i}\right)\right) x_{k} \\
& \quad+\left(1+\frac{1}{\eta}\right) \lambda_{\max }\left(E_{i}^{\mathrm{T}} P_{j} E_{i}\right)-1<0 .
\end{aligned}
$$

Noting that $1=x_{k}^{\mathrm{T}} P_{i} x_{k}$ on $\partial \varepsilon\left(P_{i}, 1\right),(23)$ is guaranteed by (19). By (18), the sufficient condition for the optimal disturbance attenuation performance index $\alpha$ is implied. This completes the proof.

Next, we show how to solve the problem by using LMIs.

Theorem 8. Consider system (1) with nonhomogeneous TP matrix (5) and let $\gamma=\alpha^{2}$ be a scalar; suppose that there exist symmetric positive definite matrices $Q_{i}=P_{i}^{-1}>0$ and $Y_{i}=$ $F_{i} Q_{i}, Z_{i}=H_{i} Q_{i}, \eta>0$, and $\lambda \in(0, \eta /(1+\eta))$, for all $i \in \Gamma$, such that

$$
\begin{gathered}
\min \gamma, \\
Q_{i}-\gamma * R^{-1}<0, \\
{\left[\begin{array}{cccc}
\left(\frac{\lambda}{\eta}-\frac{1}{1+\eta}\right) Q_{i} & * & * & * \\
\sqrt{\kappa_{i}^{1}}\left(A_{i}+B_{i}\left(D_{i} Y_{i}+D_{i}^{-} Z_{i}\right)\right) & -Q_{1} & * & * \\
\vdots & \vdots & \ddots & \vdots \\
\sqrt{\kappa_{i}^{l}}\left(A_{i}+B_{i}\left(D_{i} Y_{i}+D_{i}^{-} Z_{i}\right)\right) & * & * & -Q_{l} \\
<0, \quad \forall i \in \Gamma, j \in \pi_{j}^{k}, & & &
\end{array}\right]}
\end{gathered}
$$

$$
\begin{gathered}
{\left[\begin{array}{cc}
-\lambda & E_{i}^{T} \\
* & -Q_{k}
\end{array}\right]<0, \quad \forall i \in \Gamma, k \in \Gamma,} \\
{\left[\begin{array}{cc}
-1 & Z_{i q} \\
* & -Q_{i}
\end{array}\right]<0, \quad \forall i \in \Gamma, q \in[1, m],}
\end{gathered}
$$

where $\chi_{0}$ is a reference set and $x_{0}$ is an initial state; then the subset $\bigcap_{i=1}^{\tau} \varepsilon\left(P_{i}, 1\right)$ is the disturbance attraction domain for system (1) which satisfies an optimal disturbance attenuation performance index $\alpha$.

Proof. Denote $\gamma=\alpha^{2}$. Choose an ellipsoid $\varepsilon(R, 1)$ as a reference set. Then condition (26) can be formulated as $R / \gamma \leq P_{i}$, which is implied by (25). By applying Schur complement, it is clear that (18) and (19) follow from (25) and (26), respectively. Equation (27) implies the existence of $\lambda_{\max }$. Equation (20) is equivalent to (28). This completes the proof.

Remark 9. If we choose a polyhedron $x_{0}=\left[x_{0}^{1}, \ldots, x_{0}^{n}\right]^{\mathrm{T}}\left(x_{0}^{n}\right.$ is a point) as a reference set in Theorem 8 , then condition (22) is converted into

$$
\left[\begin{array}{cc}
-\frac{1}{\alpha^{2}} & * \\
x_{0}^{q} & -Q
\end{array}\right]<0, \quad \forall q \in[1, n] .
$$

\section{Illustrative Example}

Consider a nonhomogeneous discrete-time jump system with four modes:

$$
\begin{gathered}
A_{1}=\left[\begin{array}{cc}
0.50 & -0.30 \\
0.10 & 0.60
\end{array}\right], \quad B_{1}=\left[\begin{array}{c}
-0.026 \\
0.247
\end{array}\right], \\
E_{1}=\left[\begin{array}{l}
0.0657 \\
0.0582
\end{array}\right], \quad A_{2}=\left[\begin{array}{cc}
0.36 & -0.30 \\
0.20 & 0.50
\end{array}\right], \\
B_{2}=\left[\begin{array}{c}
-0.030 \\
0.100
\end{array}\right], \quad E_{2}=\left[\begin{array}{c}
0.0308 \\
0.0453
\end{array}\right], \\
A_{3}=\left[\begin{array}{cc}
0.70 & -0.25 \\
0.10 & 0.70
\end{array}\right], \quad B_{3}=\left[\begin{array}{c}
-0.010 \\
0.320
\end{array}\right], \\
E_{3}=\left[\begin{array}{c}
0.0236 \\
0.0292
\end{array}\right], \quad A_{4}=\left[\begin{array}{cc}
0.65 & -0.35 \\
0.25 & 0.65
\end{array}\right], \\
B_{4}=\left[\begin{array}{c}
-0.010 \\
0.220
\end{array}\right], \quad E_{4}=\left[\begin{array}{c}
0.0586 \\
0.0323
\end{array}\right] .
\end{gathered}
$$

Assume that the PDF matrix to describe the TP matrix in Table 1 is given by

$$
\pi_{N}=\left[\begin{array}{llll}
n\left(0.3, \sigma^{2}\right) & n\left(0.2, \sigma^{2}\right) & n\left(0.1, \sigma^{2}\right) & n\left(0.4, \sigma^{2}\right) \\
n\left(0.3, \sigma^{2}\right) & n\left(0.2, \sigma^{2}\right) & n\left(0.3, \sigma^{2}\right) & n\left(0.2, \sigma^{2}\right) \\
n\left(0.1, \sigma^{2}\right) & n\left(0.1, \sigma^{2}\right) & n\left(0.5, \sigma^{2}\right) & n\left(0.3, \sigma^{2}\right) \\
n\left(0.2, \sigma^{2}\right) & n\left(0.2, \sigma^{2}\right) & n\left(0.1, \sigma^{2}\right) & n\left(0.5, \sigma^{2}\right)
\end{array}\right] .
$$

Table 1 shows the obtained TP matrix with $\sigma^{2}=0.01$. 
TABLE 1: Shows the obtained TP matrix with $\sigma^{2}=0.01$.

\begin{tabular}{llll}
\hline \multicolumn{3}{c}{$\sigma^{2}=0.01$} \\
\hline 0.29917 & 0.19945 & 0.10248 & 0.39890 \\
0.29994 & 0.20006 & 0.29994 & 0.20006 \\
0.10495 & 0.10495 & 0.49381 & 0.29629 \\
0.19881 & 0.19881 & 0.10559 & 0.49679 \\
\hline
\end{tabular}

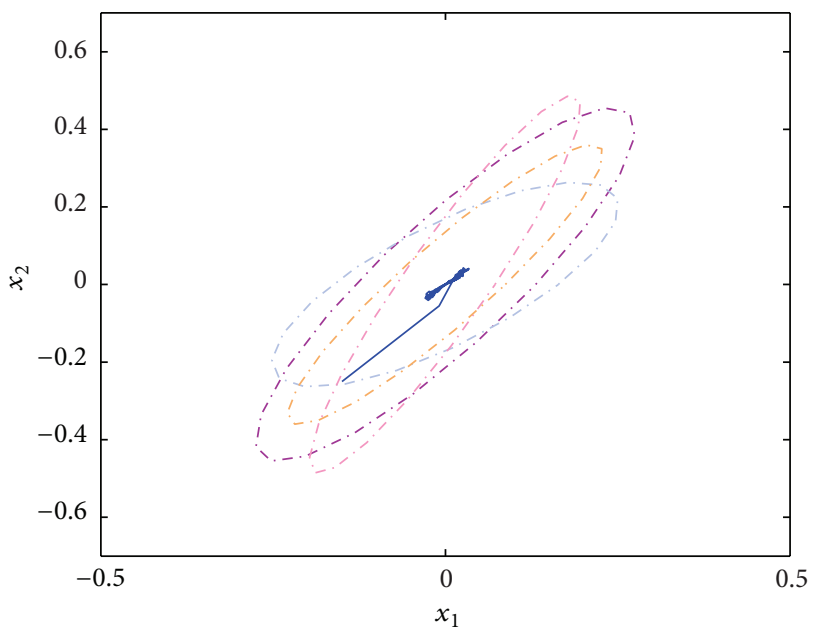

FIGURE 1: Disturbance attraction domain.

By Theorem 8 , the feedback gains are calculated as

$$
\begin{array}{ll}
F_{1}=\left[\begin{array}{ll}
2.2177 & -3.6435
\end{array}\right], & F_{2}=\left[\begin{array}{ll}
2.7909 & -6.7110
\end{array}\right], \\
F_{3}=\left[\begin{array}{ll}
3.3680 & -3.3769
\end{array}\right], & F_{4}=\left[\begin{array}{ll}
2.9303 & -4.9250
\end{array}\right] .
\end{array}
$$

Figure 1 shows a state trajectory on the boundary of the disturbance attraction domain under the bounded disturbance $w_{k}=0.5 \sin (k)$. Though the bounded disturbance exists, the state trajectory is regulated to a small neighbourhood around the origin. When the disturbance disappears, the state is driven to the origin as expected (see Figure 2), implying the stochastic stability. Figure 3 shows a trajectory of mode evolution. Table 2 shows the optimal disturbance attenuation index.

\section{Conclusions}

This paper investigated the design of the disturbance attraction domain estimation for a class of nonhomogeneous discrete-time Markov jump systems with saturation and bounded disturbance. Furthermore, the optimal disturbance attenuation index is satisfied. The numerical example shows the applicability of the results obtained as expected. The results obtained may be extended to the systems with time delay.

\section{Conflict of Interests}

The authors declare that there is no conflict of interests regarding the publication of this paper.
TABLE 2

\begin{tabular}{cccc}
\hline Parameters & $\eta^{*}$ & $\lambda^{*}$ & $\alpha_{\min }^{*}$ \\
\hline & 0.998 & 0.2510 & 0.1782 \\
\hline
\end{tabular}

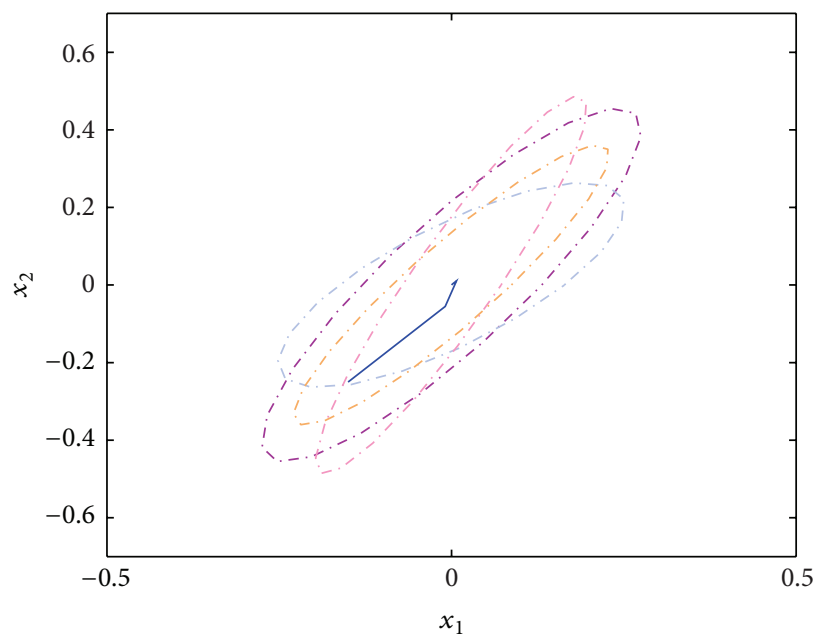

FIGURE 2: Attraction domain without disturbance.

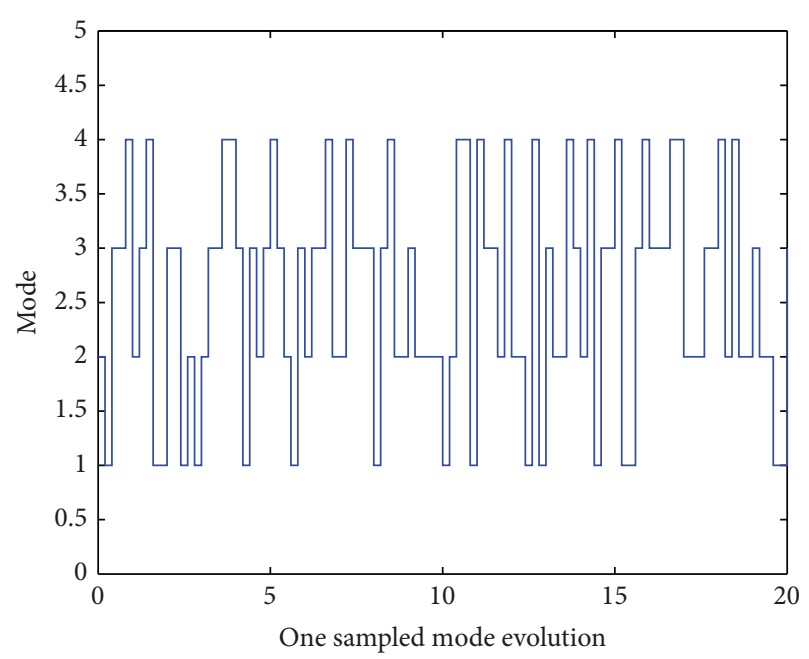

FIGURE 3: One sampled mode evolution.

\section{Acknowledgments}

This work was partially supported by National Natural Science Foundation of China (61273087), Program for Excellent Innovative Team of Jiangsu Higher Education Institutions, Jiangsu Higher Education Institutions Innovation Funds (CXZZ12_0743), the Fundamental Research Funds for the Central Universities (JUDCF12029), 111 Project (B12018), and a Discovery research grant form Australian Research Council. 


\section{References}

[1] L. Wu, X. Su, and P. Shi, "Output feedback control of Markovian jump repeated scalar nonlinear systems," IEEE Transactions on Automatic Control, vol. 59, no. 1, pp. 199-204, 2014.

[2] X. Su, P. Shi, L. Wu, and Y. Song, "A novel control design on discrete-time Takagi-Sugeno fuzzy systems with time-varying delays," IEEE Transactions on Fuzzy Systems, vol. 20, no. 6, pp. 655-671, 2013.

[3] X. Su, P. Shi, L. Wu, and M. V. Basin, "Reliable filtering with strict dissipativity for T-S fuzzy time-delay systems," IEEE Transactions on Cybernetics.

[4] F. Li, X. Wang, and P. Shi, "Robust quantized $H_{\infty}$ control for networked control systems with Markovian jumps and time delays," International Journal of Innovative Computing, Information and Control, vol. 9, no. 12, pp. 4889-4902, 2013.

[5] P. Shi and M. Liu, "On the filtering problem for continuous-time Markov jump linear systems with no observation of the Markov Chain,” European Journal of Control, vol. 17, no. 4, pp. 355-356, 2011.

[6] X. Su, P. Shi, L. Wu, and S. Nguang, "Induced $\mathrm{l}_{2}$ filtering of fuzzy stochastic systems with time-varying delays," IEEE Transactions on Cybernetics, vol. 43, no. 4, pp. 1251-1264, 2013.

[7] Z. Wu, H. Su, and J. Chu, "H-infinity filtering for singular Markovian jump systems with time delay," International Journal of Robust and Nonlinear Control, vol. 20, no. 8, pp. 939-957, 2010.

[8] L. Wu, P. Shi, H. Gao, and C. Wang, " $H_{\infty}$ filtering for 2D Markovian jump systems," Automatica, vol. 44, no. 7, pp. 1849-1858, 2008.

[9] L. Wu, P. Shi, and H. Gao, "State estimation and sliding-mode control of Markovian jump singular systems," IEEE Transactions on Automatic Control, vol. 55, no. 5, pp. 1213-1219, 2010.

[10] J. Xiong, J. Lam, H. Gao, and D. W. C. Ho, “On robust stabilization of Markovian jump systems with uncertain switching probabilities," Automatica, vol. 41, no. 5, pp. 897-903, 2005.

[11] P. Shi, Y. Xia, G. P. Liu, and D. Rees, "On designing of slidingmode control for stochastic jump systems," IEEE Transactions on Automatic Control, vol. 51, no. 1, pp. 97-103, 2006.

[12] L. Zhang and E.-K. Boukas, "Mode-dependent $H_{\infty}$ filtering for discrete-time Markovian jump linear systems with partly unknown transition probabilities," Automatica, vol. 45, no. 6 , pp. 1462-1467, 2009.

[13] L. Zhang and E.-K. Boukas, " $H_{\infty}$ control for discrete-time Markovian jump linear systems with partly unknown transition probabilities," International Journal of Robust and Nonlinear Control, vol. 19, no. 8, pp. 868-883, 2009.

[14] Y. Yin, P. Shi, and F. Liu, "Gain-scheduled robust fault detection on time-delay stochastic nonlinear systems," IEEE Transactions on Industrial Electronics, vol. 58, no. 10, pp. 4908-4916, 2011.

[15] "Internet traffic report," 2008, http://www.internettracreport .com.

[16] A. Bilbao-Guillerna, M. de la Sen, S. Alonso-Quesada, and A. Ibeas, "A multimodel scheme control for a tunnel-diode trigger circuit," in Proceedings of the IEEE Mediterranean Electrotechnical Conference, pp. 19-22, Málaga, Spain, May 2006.

[17] P. C. G. Vassiliou, "Asymptotic behavior of Markov systems," Journal of Applied Probability, vol. 19, no. 4, pp. 851-857, 1982.

[18] Y. Yin, P. Shi, F. Liu, and K. L. Teo, "Observer-based $H_{\infty}$ on nonhomogeneous Markov jump systems with nonlinear input," International Journal of Robust and Nonlinear Control, 2013.
[19] Y. Yin, P. Shi, F. Liu, and K. L. Teo, "Fuzzy model-based robust $H_{\infty}$ filtering for a class of nonlinear nonhomogeneous Markov jump systems," Signal Processing, vol. 93, no. 9, pp. 2381-2391, 2013.

[20] X. Luan, S. Zhao, and F. Liu, " $H_{\infty}$ control for discrete-time Markov jump systems with uncertain transition probabilities," IEEE Transactions on Automatic Control, vol. 58, no. 6, pp. 15661572, 2013.

[21] H. J. Sussmann, E. D. Sontag, and Y. Yang, "General result on the stabilization of linear systems using bounded controls," IEEE Transactions on Automatic Control, vol. 39, no. 12, pp. 2411-2425, 1994.

[22] H.-D. Chiang and J. S. Thorp, "Stability regions of nonlinear dynamical systems: a constructive methodology," IEEE Transactions on Automatic Control, vol. 34, no. 12, pp. 1229-1241, 1989.

[23] R. Genesio, M. Tartaglia, and A. Vicino, "On the estimation of asymptotic stability regions: state of the art and new proposals," IEEE Transactions on Automatic Control, vol. 30, no. 8, pp. 747755, 1985.

[24] T. Hu, Z. Lin, and B. M. Chen, "An analysis and design method for linear systems subject to actuator saturation and disturbance," Automatica, vol. 38, no. 2, pp. 351-359, 2002.

[25] T. Hu, Z. Lin, and B. M. Chen, "Analysis and design for discretetime linear systems subject to actuator saturation," Systems and Control Letters, vol. 45, no. 2, pp. 97-112, 2002. 


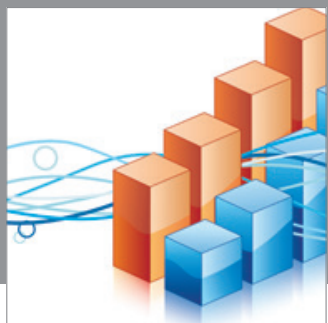

Advances in

Operations Research

mansans

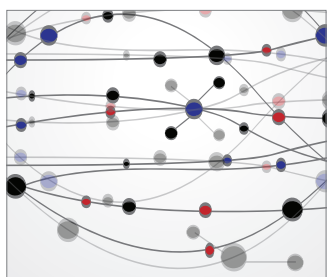

The Scientific World Journal
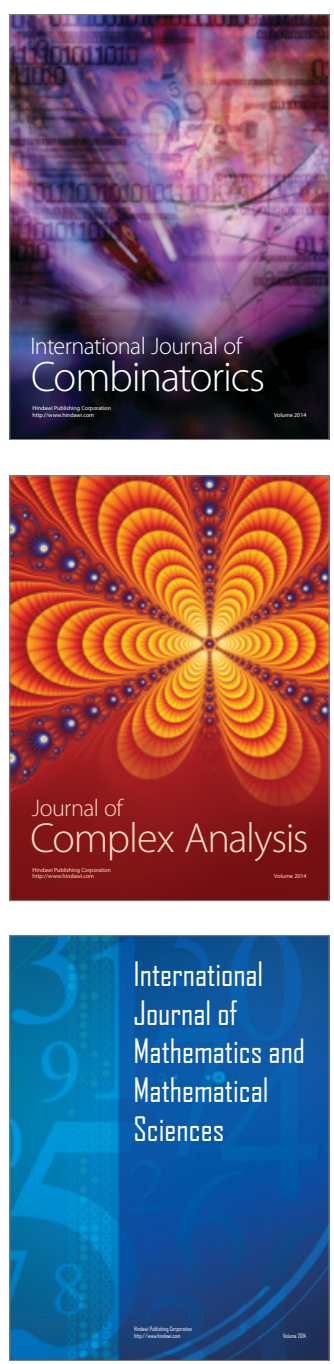
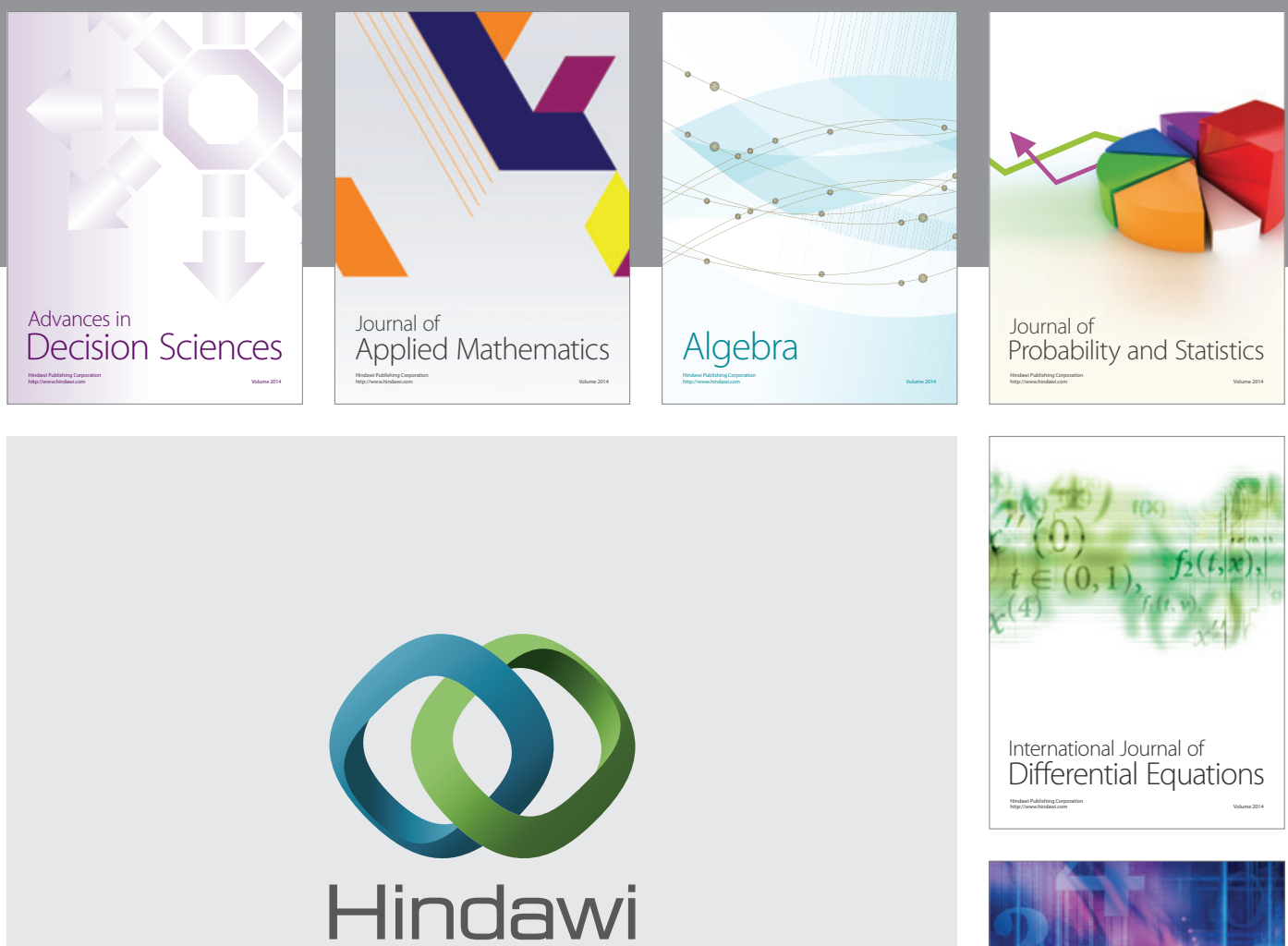

Submit your manuscripts at http://www.hindawi.com
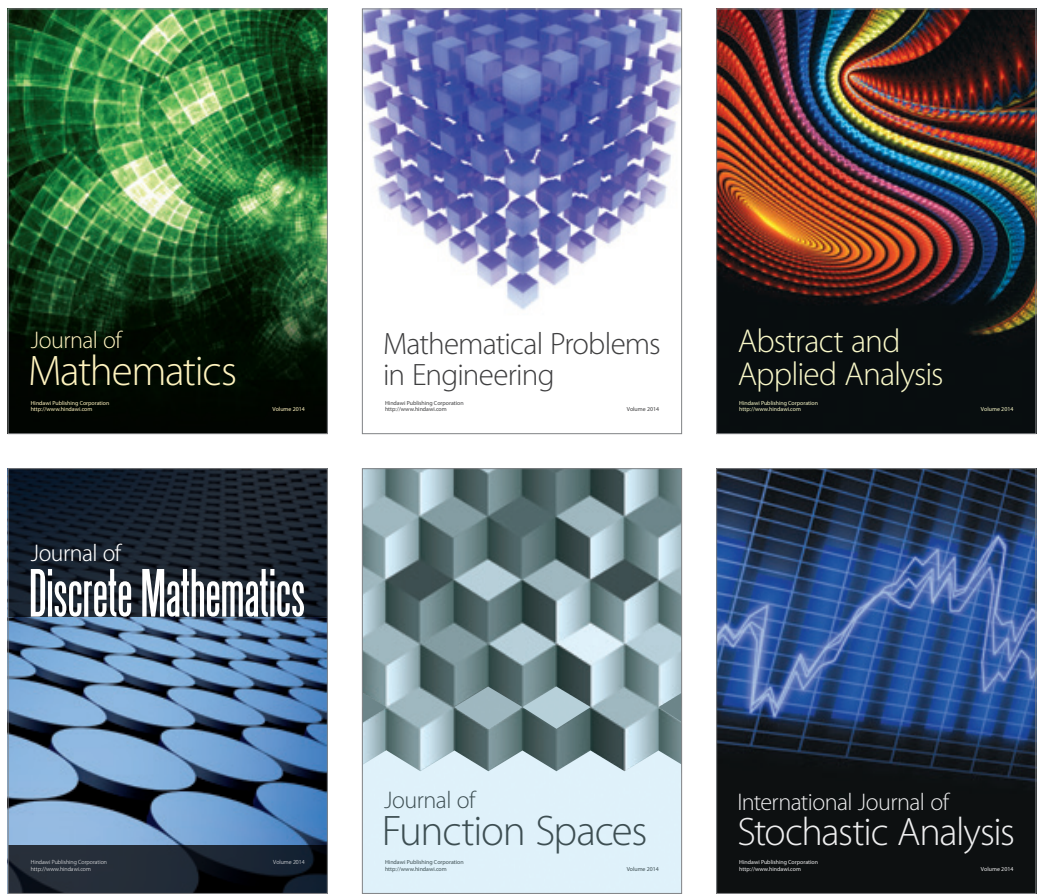

Journal of

Function Spaces

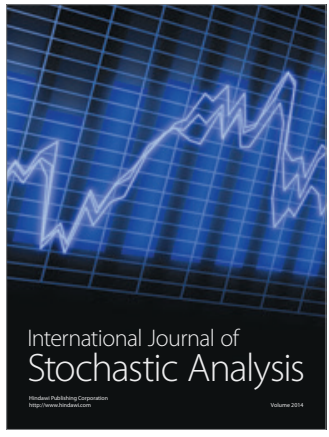

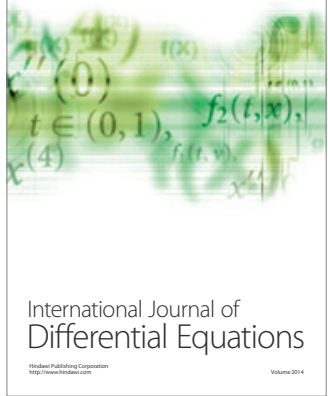
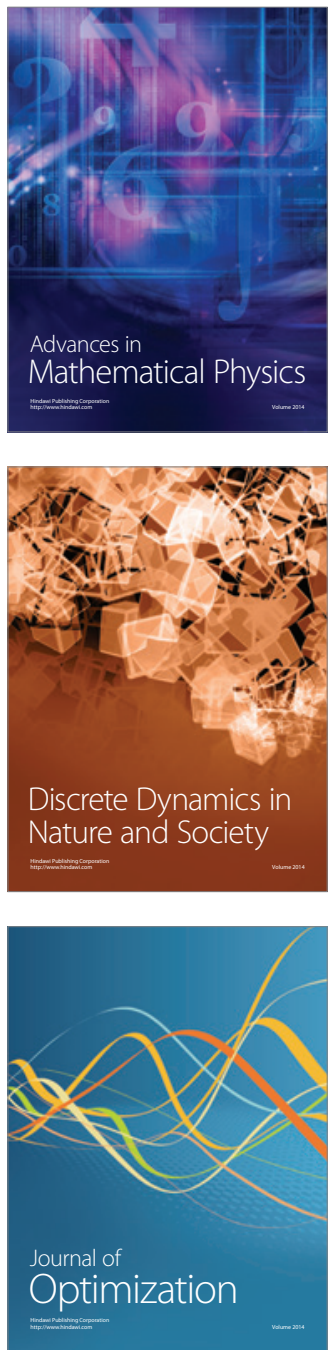